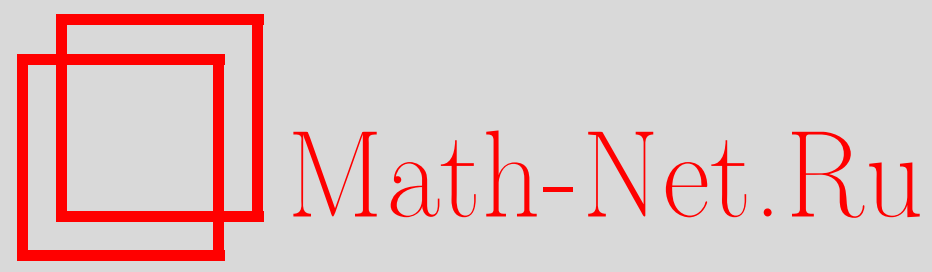

А. В. Товстолис, Приближение гладких функций на полуоси целыми функциями конечной полустепени, Матем. заметки, 2001, том 69, выпуск 6, 934-943

DOI: https://doi.org/10.4213/mzm708

Использование Общероссийского математического портала Math-Net.Ru подразумевает, что вы прочитали и согласны с пользовательским соглашением http://www.mathnet.ru/rus/agreement

Параметры загрузки:

IP : 107.22 .136 .117

26 апреля 2023 г., 16:57:40

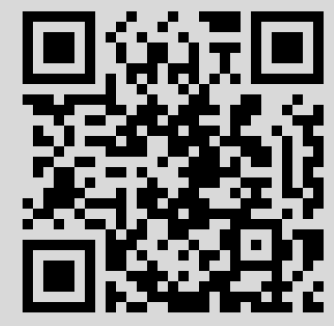




\title{
ПРИБЛИЖЕНИЕ ГЛАДКИХ ФУНКЦИЙ НА ПОЛУОСИ ЦЕЛЫМИ ФУНКЦИЯМИ КОНЕЧНОЙ ПОЛУСТЕПЕНИ
}

\section{А. В. Товстолис}

\begin{abstract}
В статье изучается поточечное приближение функции, заданной на вещественной полуоси и имеющей там почти всюду ограниченную $r$-ю производную. В качестве аппарата приближения используются введенные Бернштейном целые функции конечной полустепени. В работе получена асимптотически точная оценка поточечного приближения для указанного класса.

Библиографория: 11 названий.
\end{abstract}

Пусть $E$ - замыкание непустого открытого подмножества $\mathbb{R}$. Через $W^{r}(E), r \in \mathbb{N}$, обозначают класс функций $f$, у которых производная $f^{(r-1)}$ абсолютно непрерьвна на любом интервале, содержашемся в $E$, и $\left|f^{(r)}\right| \leqslant 1$ почти всюду (в смысле меры Лебега) на $E$.

Аппроксимация функций из этих классов изучалась во многих работах (см. [1]-[4] с обширной библиографией). В настоящей работе рассматривается случай $E=\mathbb{R}_{+}=$ $[0,+\infty)$. Естественным аппаратом приближения здесь являются введенные Бернштейном целые функции конечной полустепени, т.е. целые функции порядка $1 / 2$ нормального типа (см. [5]). В этом случае равномерная на $\mathbb{R}_{+}$апроксимация не всегда возможна (см. [5, с. 481]) и для получения прямых и обратных теорем используется поточечное приближение. Такие теоремы были получены Брудньм [6] (см. также [2]).

Вопрос о точной константе в главном члене поточечной оценки скорости приближения функции класса $W^{r}$ на различных множествах рассматривается, начиная с работы Никольского [7] (см. также работы Корнейчука и Половины [8], Темлякова [9] и др.). В случае отрезка, где изучается апшроксимация алгебраическими многочленами, поточечная оценка скорости приближения с точной константой в главном члене недавно получена Тригубом [10].

В данной работе получено решение этой задачи на $\mathbb{R}_{+}$. Основным результатом является теорема 1 (см. ниже), которая дает асимптотически точную оценку скорости приближения функции класса $W^{r}\left(\mathbb{R}_{+}\right)$целыми функциями конечной полустепени.

1. Формулировка основного результата. Далее, как обычно, для $\sigma>0$ через $\mathbf{H}_{\sigma}$ обозначается класс цельх функций конечной полустепени типа не вьше $\sigma$, т.е. класс целых функций $f$ таких, что

$$
f(z)=\sum_{k=0}^{\infty} \frac{a_{k} z^{k}}{(2 k) !}
$$


где

$$
\varlimsup_{k \rightarrow \infty} \sqrt[2 k]{\left|a_{k}\right|} \leqslant \sigma
$$

или, что то же самое, функция $F(z)=f\left(z^{2}\right)$ является целой функцией экспоненциального типа не выше $\sigma$ (см. [5, с. 479]). Пусть также

$$
K_{r}=\frac{4}{\pi} \sum_{k=0}^{\infty} \frac{(-1)^{k(r+1)}}{(2 k+1)^{r+1}}
$$

Имеет место следующая

Tеорема 1. Пусть $f \in W^{r}\left(\mathbb{R}_{+}\right), r \in \mathbb{N}$. Тогда для любого $\sigma>0$ найдется функиия $g \in \mathbf{H}_{\sigma}$, для которой

$$
|f(x)-g(x)| \leqslant \frac{2^{r} K_{r}}{\sigma^{r}} x^{r / 2}+\frac{\gamma}{\sigma^{r+1}} x^{(r-1) / 2} \quad \forall x \geqslant 0,
$$

әде $\gamma$ - некоторая положительная константа, зависящая лишь от $r$.

При этом константу $2^{r} K_{r}$ в (1), вообще говоря, уменьшить нельзя.

Как и в работе [10], эта теорема доказывается без оценок интегральных операторов приближения, как это обычно делается при доказательстве такого рода утверждений (см., например, [1], [2]).

Автор выражает глубокую благодарность профессору Р. М. Тригубу за постановку задачи и внимание к работе.

2. Вспомогательные утверждения. Введем некоторые обозначения. Если $0 \in E$, то $W_{0}^{r}(E)=\left\{f \in W^{r}(E): f^{(\nu)}(0)=0, \nu \in[1, r-1]\right\}$. Для произвольной константы $\alpha>0$ положим $\alpha W^{r}(E)=\left\{f: f / \alpha \in W^{r}(E)\right\}$. Аналогично определяется класс $\alpha W_{0}^{r}(E)$. Как обычно, для $\sigma>0$ через $\mathbf{E}_{\sigma}$ обозначается класс целых функций экспоненциального типа не вьше $\sigma ; \mathbf{B}_{\sigma}=\mathbf{E}_{\sigma} \cap L_{\infty}(\mathbb{R})$ - класс Бернштейна;

$$
\mathscr{A}_{\sigma}(f)=\inf _{g \in \mathbf{E}_{\sigma}}\|f-g\|_{C(\mathbb{R})}
$$

$[t]$ - целая часть числа $t \in \mathbb{R}$. Через $\gamma(a, b)$ будем обозначать положительныеконстанты, зависящие лиш от $a$ и $b$, в разных случаях, вообще говоря, различные.

Лемма 1. Пусть $f \in W_{0}^{r}\left(\mathbb{R}_{+}\right), r \in \mathbb{N}$. Тогда

а) найдутся функиии $\varphi_{r} \in W_{0}^{r}(\mathbb{R})$ и $\psi_{r} \in \gamma(r) W_{0}^{r+1}(\mathbb{R})$ такие, что

$$
f\left(x^{2}\right)=(2 x)^{r} \varphi_{r}(x)+(2 x)^{r-1} \psi_{r}(x) \quad \forall x \in \mathbb{R}
$$

$u$

$$
\varphi_{r}^{(r)}(x)=f^{(r)}\left(x^{2}\right) \quad \text { для почти всех } x \in \mathbb{R} ;
$$

кроме того, функиии $x^{r} \varphi_{r}(x)$ и $x^{r-1} \psi_{r}(x)$ четные;

б) найдутся функиии $\xi_{r} \in W_{0}^{r}\left(\mathbb{R}_{+}\right)$u $\zeta_{r} \in \gamma(r) W_{0}^{r+1}(\mathbb{R})$ такие, что

$$
2^{r}|x|^{r} f(|x|)=\xi_{r}\left(x^{2}\right)+(2 x)^{r-1} \zeta_{r}(x) \quad \forall x \in \mathbb{R} .
$$


ДокАЗАТЕЛЬСТВо. а) При $r=1$ достаточно положить

$$
\varphi_{1}(x)=\int_{0}^{x} f^{\prime}\left(t^{2}\right) d t, \quad \psi_{1}(x)=-2 \int_{0}^{x} \varphi_{1}(t) d t
$$

и $\gamma(1)=2$.

Далее по индукции. Пусть лемма верна для данного $r \in \mathbb{N}$. Докажем ее для $r+1$. Пусть $f \in W_{0}^{r+1}\left(\mathbb{R}_{+}\right)$, тогда $f^{\prime} \in W_{0}^{r}\left(\mathbb{R}_{+}\right)$. По предположению индукции для любого $x \in \mathbb{R}$

$$
f^{\prime}\left(x^{2}\right)=(2 x)^{r} \varphi_{r}(x)+(2 x)^{r-1} \psi_{r}(x),
$$

где $\varphi_{r} \in W_{0}^{r}(\mathbb{R}), \varphi_{r}^{(r)}(x)=f^{(r+1)}\left(x^{2}\right)$ для почти всех $x \in \mathbb{R}$ и $\psi_{r} \in \gamma(r) W_{0}^{r+1}(\mathbb{R})$. Умножая это тождество на $2 x$ и интегрируя, получим

$$
f\left(x^{2}\right)=\int_{0}^{x}(2 t)^{r+1} \varphi_{r}(t) d t+\int_{0}^{x}(2 t)^{r} \psi_{r}(t) d t .
$$

Пусть

$$
\varphi_{r+1}(x)=\int_{0}^{x} \varphi_{r}(t) d t
$$

тогда $\varphi_{r+1} \in W_{0}^{r+1}(\mathbb{R})$ и $\varphi_{r+1}^{(r+1)}(x)=\varphi_{r}^{(r)}(x)=f^{(r+1)}\left(x^{2}\right)$ для почти всех $x \in \mathbb{R}$. После интегрирования по частям из (5) имеем

$$
f\left(x^{2}\right)=(2 x)^{r+1} \varphi_{r+1}(x)+\int_{0}^{x}(2 t)^{r} G_{r+1}(t) d t,
$$

где $G_{r+1}(t)=\psi_{r}(t)-2(r+1) \varphi_{r+1}(t)$. Нетрудно видеть, что

$$
G_{r+1} \in \gamma_{1}(r+1) W_{0}^{r+1}(\mathbb{R})
$$

где $\gamma_{1}(r+1)=\gamma(r)+2(r+1)$.

Положим

$$
G_{r+\nu+1}(x)=\int_{0}^{x} G_{r+\nu}(t) d t, \quad \nu \in \mathbb{N}
$$

и

$$
\psi_{r+1}(x)=\frac{1}{(2 x)^{r}} \int_{0}^{x}(2 t)^{r} G_{r+1}(t) d t=\sum_{\nu=0}^{r} \frac{(-1)^{\nu} r !}{(r-\nu) !} \frac{G_{r+\nu+2}(x)}{x^{\nu}} .
$$

По формуле Лейбнища

$$
\psi_{r+1}^{(s)}(x)=G_{r+2}^{(s)}(x)+\sum_{\nu=1}^{r} \frac{(-1)^{\nu} r !}{(r-\nu) !}\left(\sum_{k=0}^{s}\left(\begin{array}{l}
s \\
k
\end{array}\right) \frac{(-1)^{k}(\nu+k-1) !}{(\nu-1) !} \frac{G_{r+\nu+2}^{(s-k)}(x)}{x^{\nu+k}}\right)
$$

для $s \in[0, r+2]$. Очевидно, что при любьх $\nu \in[0, r]$ и $m \in[0, r+\nu+2]$ вьполнены соотношения

$$
G_{r+\nu+2}^{(m)}(x)= \begin{cases}G_{r+\nu+2-m}(x), & m \in[0, \nu+1] \\ G_{r+1}^{(m-\nu-1)}(x), & m \in[\nu+2, r+\nu+2] .\end{cases}
$$


Так как $G_{r+1}^{(s)}(0)=0$ при $s \in[0, r]$, получаем $G_{r+\nu+2}^{(m)}(0)=0$ при любом $m \in[0, r+\nu+1]$. Таким образом, $G_{r+\nu+2}^{(s-k+l)}(0)=0$ при $l \in[0, \nu+k-1](\nu \in \mathbb{N}, s \in[0, r+2], k \in[0, s])$.

Теперь из (10) находим, что

$$
\psi_{r+1}^{(s)}(0)=0 \quad \text { при } s \in[0, r+1] \text { и }\left\|\psi_{r+1}^{(r+2)}\right\|_{\infty} \leqslant \gamma_{2}(r+1)\left\|G_{r+2}^{(r+2)}\right\|_{\infty},
$$

где

$$
\gamma_{2}(r+1)=1+\sum_{\nu=1}^{r} \frac{r !}{(r-\nu) !}\left(\sum_{k=0}^{r+2}\left(\begin{array}{c}
r+2 \\
k
\end{array}\right) \frac{1}{(\nu-1) !(\nu+k)}\right) .
$$

Из (7) и (8) следует, что

$$
\left\|G_{r+2}^{(r+2)}\right\|_{\infty}=\left\|G_{r+1}^{(r+1)}\right\|_{\infty} \leqslant \gamma_{1}(r+1) .
$$

Таким образом, $\psi_{r+1} \in \gamma(r+1) W_{0}^{r+2}(\mathbb{R})$, где $\gamma(r+1)=\gamma_{1}(r+1) \gamma_{2}(r+1)$, и из $(6)$ и (8) получаем (2).

Четность функций $x^{r} \varphi_{r}(x)$ и $x^{r-1} \psi_{r}(x)$ следует из (2) и (3).

б) Покажем, что найдутся функции $\Phi_{r} \in W_{0}^{r}\left(\mathbb{R}_{+}\right)$и $\Psi_{r}$, удовлетворяющая условию $\Psi_{r}\left(x^{2}\right) \in \gamma(r) W_{0}^{r+1}(\mathbb{R})$, такие, что для любого $x \geqslant 0$ имеет место равенство

$$
(2 \sqrt{x})^{r} f(\sqrt{x})=\Phi_{r}(x)+(2 \sqrt{x})^{r-1} \Psi_{r}(x) .
$$

При $r=1$ достаточно положить

$$
\Phi_{1}(x)=\int_{0}^{x} f^{\prime}(\sqrt{t}) d t, \quad \Psi_{1}(x)=\int_{0}^{x} \frac{f(\sqrt{t})}{\sqrt{t}} d t, \quad x \geqslant 0,
$$

и $\gamma(1)=2$.

Далее нужно воспользоваться методом математической индукции и повторить рассуждения, аналогичные проведенным при доказательстве части а).

Для доказательства (4) теперь осталось заменить $x$ на $x^{2}$ в (11) и положить

$$
\xi_{r}(x)=\Phi_{r}(x) \quad \forall x \geqslant 0, \quad \zeta_{r}(x)=(\operatorname{sign} x)^{r-1} \Psi_{r}\left(x^{2}\right) \quad \forall x \in \mathbb{R} .
$$

Лемма 2. Пусть $f \in \mathbf{E}_{\sigma}$ и существуют неотрицательные константы $A$ и $B$ такие, что $|f(x)| \leqslant A|x|+B$ для любого $x \in \mathbb{R}$. Тогда

а) имеет место оценка

$$
\left|f^{\prime}(x)\right| \leqslant C \sigma|x|+C \quad \forall x \in \mathbb{R}
$$

əde

$$
C=\max \left\{\max _{|y| \leqslant 1}\left|\frac{f(y)-f(0)}{y}\right|, \quad A+2 B\right\} ;
$$

б) для всех $x \in \mathbb{R}, m \in \mathbb{N}, T>0$ имеет место неравенство

$$
\left|\xi_{m}(x)-\xi_{m}(0)\right| \leqslant|x| \cdot \max \left\{\frac{C \sigma}{T}+C \sigma+\frac{C}{T}, \quad \frac{A}{T}+2 A+\frac{2 B}{T}\right\},
$$

əде $\xi_{m}(x)=(f(x+m T)+f(x-m T)) /(2 m T)$. 
ДоказАТЕЛЬСтво. а) Из условия следует, что функция $F(x)=(f(x)-f(0)) / x$ принадлежит классу $\mathbf{E}_{\sigma}$ и $|F(x)| \leqslant C$ при любом $x \in \mathbb{R}$. Таким образом, $F \in \mathbf{B}_{\sigma}$. Используя неравенство Бернштейна (см., например, $[1$, гл. IV , п. 84 , теорема 1$]$ ), получаем

$$
\left|F^{\prime}(x)\right| \leqslant C \sigma \quad \forall x \in \mathbb{R} .
$$

Из определения $F(x)$ имеем также

$$
F^{\prime}(x)=\frac{f^{\prime}(x)-F(x)}{x}
$$

откуда

$$
\left|f^{\prime}(x)\right| \leqslant C \sigma|x|+C \quad \forall x \in \mathbb{R} .
$$

б) Из определения $\xi_{m}$ следует, что для всех $x \in \mathbb{R}, m \in \mathbb{N}$ выполнены неравенства

$$
\left|\xi_{m}(x)\right| \leqslant \frac{A}{2 m T}(|x+m T|+|x-m T|)+\frac{B}{m T} \leqslant \frac{A}{T}|x|+A+\frac{B}{T} .
$$

Кроме того, $\xi_{m}^{\prime}(x)=\left(f^{\prime}(x+m T)+f^{\prime}(x-m T)\right) /(2 m T)$. Поэтому, применяя (12) к функции $f$, получим

$$
\begin{aligned}
\left|\xi_{m}^{\prime}(x)\right| & \leqslant \frac{1}{2 m T}(C \sigma|x+m T|+C \sigma|x-m T|+2 C) \\
& \leqslant \frac{C \sigma}{T}|x|+C \sigma+\frac{C}{T} \quad \forall x \in \mathbb{R}, \quad m \in \mathbb{N} .
\end{aligned}
$$

Используя теорему Лагранжа о среднем, отсюда имеем

$$
\left|\xi_{m}(x)-\xi_{m}(0)\right| \leqslant\left(\frac{C \sigma}{T}+C \sigma+\frac{C}{T}\right)|x| \quad \forall m \in \mathbb{N}, \quad|x| \leqslant 1 .
$$

Кроме того, из (13) следует

$$
\left|\xi_{m}(x)-\xi_{m}(0)\right| \leqslant\left(\frac{A}{T}+2 A+\frac{2 B}{T}\right)|x| \quad \forall m \in \mathbb{N}, \quad|x|>1 .
$$

Из оценок (14) и (15) получаем утверждение б).

Лемма 3. Пусть $f \in W^{r}(\mathbb{R})$ при некотором $r \in \mathbb{N}$. Тогда для любого $\varepsilon \in(0,1]$ найдется набор комплексных чисел $\left\{a_{k, \varepsilon}\right\}_{k=0}^{r-1}$ такой, что функиия

$$
\mathscr{F}_{\varepsilon}(x)=f(x)-\sum_{k=0}^{r-1} a_{k, \varepsilon} \sin ^{k} \varepsilon x
$$

будет принадлежсать классу $\left(1+\varepsilon \gamma_{0}(f, r)\right) W_{0}^{r}(\mathbb{R})$ с некоторой положительной константой $\gamma_{0}(f, r)$, не зависящей от $\varepsilon$.

Кроме того, если функция $f$ является четной (или нечетной), то и функция $\mathscr{F}_{\varepsilon}$ такая же. 
ДокАЗАТЕЛЬСтво. Рассмотрим систему линейных уравнений

$$
\frac{1}{\varepsilon^{\nu}} f^{(\nu)}(0)-\sum_{k=0}^{\nu} a_{k, \varepsilon}\left(\sin ^{k} x\right)^{(\nu)}(0)=0, \quad \nu \in[0, r-1],
$$

относительно неизвестных $a_{k, \varepsilon}$.

Поскольку $\left(\sin ^{\nu} x\right)^{(\nu)}(0)=\nu ! \neq 0$, то матрица данной системы невырождена, следовательно, система (16) имеет единственное решение. При этом каждое из $a_{k, \varepsilon}$ является линейной комбинацией выражений $f(0), f^{\prime}(0) / \varepsilon, \ldots, f^{(k)}(0) / \varepsilon^{k}$, поэтому найдется константа $c(f, k)>0$ такая, что

$$
\left|a_{k, \varepsilon}\right| \leqslant \frac{c(f, k)}{\varepsilon^{k}} \quad \forall k \in[0, r-1], \quad \varepsilon \in(0,1] .
$$

Далее, для всех $k \in \mathbb{Z}_{+}$имеет место разложение

$$
\left(\sin ^{k} x\right)^{(r)}=\left(\frac{i}{2}\right)^{k} \sum_{\nu=0}^{k}\left(\begin{array}{l}
k \\
\nu
\end{array}\right)(-1)^{\nu}(i(2 \nu-k))^{r} e^{i(2 \nu-k) x},
$$

откуда

$$
\left|\left(\sin ^{k} \varepsilon x\right)^{(r)}\right| \leqslant \frac{\varepsilon^{r}}{2^{k}} \sum_{\nu=0}^{k}\left(\begin{array}{l}
k \\
\nu
\end{array}\right)|2 \nu-k|^{r} .
$$

Из полученных оценок и определения $\mathscr{F}_{\varepsilon}$ следует утверждение леммы 3.

Для доказательства точности константы $2^{r} K_{r}$ в главном члене неравенства (1) нам потребуется следующее утверждение.

Лемма 4. Пусть $r \in \mathbb{N}$ и существуют константы $A_{r} u \gamma_{1}(r)$ такие, ито для любой функиии $F \in W^{r}\left(\mathbb{R}_{+}\right)$и любого $\sigma>0$ найдется функиия $G \in \mathbf{H}_{\sigma}$, удовлетворяющая неравенству

$$
|F(x)-G(x)| \leqslant \frac{A_{r}}{\sigma^{r}} x^{r / 2}+\frac{\gamma_{1}(r)}{\sigma^{r+1}} x^{(r-1) / 2} \quad \forall x \geqslant 0 .
$$

Тогда для любой периодической функиии $f \in W^{r}(\mathbb{R})$, удовлетворяющей условию $f(-x)=(-1)^{r-1} f(x), x \in \mathbb{R}$, при любом $\sigma>0$ выполняется неравенство

$$
\mathscr{A}_{\sigma}[f] \leqslant \frac{A_{r}}{2^{r} \sigma^{r}} .
$$

ДокАЗАТЕЛЬСтво. Пусть $\sigma>0$ и $k \in \mathbb{N}$,

$$
k \geqslant \frac{2 \pi}{T} \max \left\{1, \frac{r-1}{\sigma}\right\}
$$

Рассмотрим функцию

$$
\mathscr{F}(x)=\frac{\mathscr{F}_{\varepsilon}(x)}{1+\varepsilon \gamma_{0}(f, r)}=\frac{1}{1+\varepsilon \gamma_{0}(f, r)}\left(f(x)-\sum_{\nu=0}^{r-1} a_{\nu, \varepsilon} \sin ^{\nu} \varepsilon x\right),
$$


где $\varepsilon=2 \pi /(k T)$ (здесь функция $\mathscr{F}_{\varepsilon}(x)$ и константа $\gamma_{0}(f, r)$ - из леммы 3$)$.

Из условия леммы 4 и леммы 3 получаем, что $\mathscr{F} \in W_{0}^{r}(\mathbb{R})$ и

$$
\mathscr{F}(-x)=(-1)^{r-1} \mathscr{F}(x) \quad \forall x \in \mathbb{R}
$$

Кроме того, очевидно, что функция $\mathscr{F}$ - периодическая с периодом $k T$. Из (18) имеем также

$$
|x|^{r} \mathscr{F}(|x|)=x^{r-1}|x| \mathscr{F}(x) \quad \forall x \in \mathbb{R}
$$

Из утверждения б) леммы 1 следует, что

$$
2^{r} x^{r-1}|x| \mathscr{F}(x)=\xi_{r}\left(x^{2}\right)+(2 x)^{r-1} \zeta_{r}(x) \quad \forall x \in \mathbb{R}
$$

где $\xi_{r} \in W_{0}^{r}\left(\mathbb{R}_{+}\right), \zeta_{r} \in \gamma(r) W_{0}^{r+1}(\mathbb{R})$. Отсюда

$$
\lim _{x \rightarrow 0} \frac{\xi_{r}\left(x^{2}\right)}{x^{r-1}}=0
$$

В силу прямой теоремы о приближении цельми функциями экспоненциального типа не вьше $\sigma$ (см., например, [1, гл. V , п. 101, теорема 1] и обсуждение в п. 99 там же или $[2$, гл. $\mathrm{V}, \S 5.6])$ найдется функция $g_{\sigma, r} \in \mathbf{E}_{\sigma}$ такая, что

$$
\left|\zeta_{r}(x)-g_{\sigma, r}(x)\right| \leqslant \frac{\gamma(r) K_{r+1}}{\sigma^{r+1}} \quad \forall x \in \mathbb{R}
$$

Кроме того, по условию леммы существует функция $G \in \mathbf{H}_{\sigma}$ такая, что

$$
\left|\xi_{r}\left(x^{2}\right)-G\left(x^{2}\right)\right| \leqslant \frac{A_{r}}{\sigma^{r}}|x|^{r}+\frac{\gamma_{1}(r)}{\sigma^{r+1}}|x|^{r-1} \quad \forall x \in \mathbb{R} .
$$

Из этого неравенства и (20) следует, что существует и конечен $\lim _{x \rightarrow 0}\left(G\left(x^{2}\right) / x^{r-1}\right)$, поэтому

$$
\frac{G\left(x^{2}\right)}{x^{r-1}} \in \mathbf{E}_{\sigma}
$$

Теперь из (19), (21) и (22) вытекает

$$
|| x\left|\mathscr{F}(x)-G_{\sigma}(x)\right| \leqslant \frac{A_{r}}{2^{r} \sigma^{r}}|x|+\frac{\gamma_{1}(r)+2^{r-1} \gamma(r) K_{r+1}}{2^{r} \sigma^{r+1}} \quad \forall x \in \mathbb{R}
$$

где

$$
G_{\sigma}(x)=\frac{G\left(x^{2}\right)}{2^{r} x^{r-1}}+\frac{1}{2} g_{\sigma, r}(x) \in \mathbf{E}_{\sigma} .
$$

Поскольку функция $\mathscr{F} \in W_{0}^{r}(\mathbb{R})$ периодическая, то $\mathscr{F} \in L_{\infty}(\mathbb{R})$ и из $(23)$ имеем

$$
\left|G_{\sigma}(x)\right| \leqslant\left(\frac{A_{r}}{2^{r} \sigma^{r}}+\|\mathscr{F}\|_{\infty}\right)|x|+\frac{\gamma_{1}(r)+2^{r-1} \gamma(r) K_{r+1}}{2^{r} \sigma^{r+1}} \quad \forall x \in \mathbb{R}
$$


Записьвая (23) для $x+m k T$ и $x-m k T$, складьвая полученные неравенства и учитывая, что $|x+m k T|+|x-m k T|=2 m k T$ при $|x| \leqslant m k T$, получаем

$$
\begin{gathered}
\left|\mathscr{F}(x)-\frac{G_{\sigma}(x+m k T)+G_{\sigma}(x-m k T)}{2 m k T}\right| \leqslant \frac{A_{r}}{2^{r} \sigma^{r}}+\frac{\gamma_{1}(r)+2^{r-1} \gamma(r) K_{r+1}}{2^{r} \sigma^{r+1} m k T} \\
\forall x:|x| \leqslant m k T .
\end{gathered}
$$

Из (24) и утверждения б) леммы 2 следует, что найдутся функция $g_{\sigma} \in \mathbf{E}_{\sigma}$ и возрастающая последовательность $\left\{m_{l}\right\}$ натуральных чисел такие, что при $l \rightarrow \infty$

$$
\frac{G_{\sigma}\left(x+m_{l} k T\right)+G_{\sigma}\left(x-m_{l} k T\right)}{2 m_{l} k T} \rightarrow g_{\sigma}(x)
$$

равномерно на любом компакте из $\mathbb{R}$. Полагая в $(25) m=m_{l}$, при $l \rightarrow \infty$ имеем

$$
\left|\mathscr{F}(x)-g_{\sigma}(x)\right| \leqslant \frac{A_{r}}{2^{r} \sigma^{r}} \quad \forall x \in \mathbb{R} .
$$

Это означает, что

$$
\left|f(x)-\Theta_{\sigma}(x)\right| \leqslant \frac{A_{r}}{2^{r} \sigma^{r}}\left(1+\varepsilon \gamma_{0}(f, r)\right) \quad \forall x \in \mathbb{R},
$$

где

$$
\Theta_{\sigma}(x)=\left(1+\varepsilon \gamma_{0}(f, r)\right) g_{\sigma}(x)+\sum_{\nu=0}^{r-1} a_{\nu, \varepsilon} \sin ^{\nu} \varepsilon x .
$$

Из условия (17) и определения $\varepsilon$ видно, что $\Theta_{\sigma} \in \mathbf{E}_{\sigma}$. Тогда из (26) следует, что

$$
\mathscr{A}_{\sigma}[f] \leqslant \frac{A_{r}}{2^{r} \sigma^{r}}\left(1+\frac{2 \pi}{k T} \gamma_{0}(f, r)\right) \text {. }
$$

Переходя в этом неравенстве к пределу при $k \rightarrow \infty$, получаем утверждение леммы 4 . Нам потребуются также некоторые свойства идеальных сплайнов Эйлера.

ЛЕМма 5. Пусть

$$
\varphi_{\lambda, r}(x)=\frac{4}{\pi \lambda^{r}} \sum_{k=0}^{\infty} \frac{\sin ((2 k+1) \lambda x-\pi r / 2)}{(2 k+1)^{r+1}}, \quad r \in \mathbb{Z}_{+}, \quad x \in \mathbb{R}, \quad \lambda>0
$$

- идеальные сплайны Эйлера (см., например, [3, гл. 3, § 3.1]). Для произвольных $\sigma>0$ и $r \in \mathbb{Z}_{+}$функиии $\Phi_{\sigma, r}(x)=\varphi_{[\sigma]+1, r}(x)$ обладают следующими свойствами:

1) $\Phi_{\sigma, r}-$ периодическая с периодом $2 \pi$;

2) $\Phi_{\sigma, r}(-x)=(-1)^{r-1} \Phi_{\sigma, r}(x)$ для всех $x \in \mathbb{R}$;

3) при любых $j \in[0, r]$ выполнено равенство $\left\|\Phi_{\sigma, r}^{(j)}\right\|_{\infty}=K_{r-j} /([\sigma]+1)^{r-j}$;

4) $\mathscr{A}_{\sigma}\left[\Phi_{\sigma, r}\right]=K_{r} /([\sigma]+1)^{r}$. 
ДокАЗАТЕЛЬСтво. Свойства 1) и 2) следуют непосредственно из определения функции $\Phi_{\sigma, r}$. Свойство 3$)$ доказано в $[3$, гл. $3, \S 3.1]$.

Для доказательства свойства 4) применим теорему Бернштейна [11, теорема IV], согласно которой если $f$ - непрерывна и ограничена на всей числовой оси и в точках некоторого полного множества степени $\sigma$ (см. [11], а также [2, гл. II, $\S 2.12$, п. 2.12.4]) вьполнено неравенство $(-1)^{k} f\left(x_{k}\right) \geqslant \mu>0$, то $\mathscr{A}_{\sigma}[f] \geqslant \mu$.

Как известно (см. [2, гл. II, § 2.12, п. 2.12.4]), примером такого множества точек может служить последовательность $x_{k}=k \pi /([\sigma]+1)+\alpha, k \in \mathbb{Z}$.

Поэтому, полагая

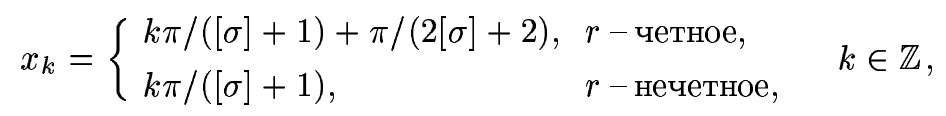

получим

$$
(-1)^{k} \Phi_{\sigma, r}\left(x_{k}\right)=\left\{\begin{array}{ll}
(-1)^{r / 2} K_{r} /([\sigma]+1)^{r}, & r \text {-четное, } \\
(-1)^{[r / 2]+1} K_{r} /([\sigma]+1)^{r}, & r \text {-нечетное, }
\end{array} \quad k \in \mathbb{Z} .\right.
$$

Теперь из приведенной теоремы Бернштейна и свойства 3) имеем

$$
\mathscr{A}_{\sigma}\left[\Phi_{\sigma, r}\right]=\left\|\Phi_{\sigma, r}\right\|_{\infty}=\frac{K_{r}}{([\sigma]+1)^{r}}
$$

3. Доказательство теоремы 1. Рассмотрим функцию

$$
F(x)=f(x)-\sum_{k=0}^{r-1} \frac{1}{k !} f^{(k)}(0) x^{k}
$$

Ясно, что $F^{(r)} \equiv f^{(r)}$ и функция $F$ удовлетворяет условиям леммы 1. Из $(2)$ получаем

$$
F\left(x^{2}\right)=(2 x)^{r} \varphi_{r}(x)+(2 x)^{r-1} \psi_{r}(x) \quad \forall x \in \mathbb{R},
$$

где $\varphi_{r} \in W_{0}^{r}(\mathbb{R}), \psi_{r} \in \gamma_{1}(r) W_{0}^{r+1}(\mathbb{R})$ и, кроме того, функции $x^{r} \varphi_{r}(x)$ и $x^{r-1} \psi_{r}(x)-$ четные.

В силу прямой теоремы о приближении цельми функциями экспоненциального типа (см. $[1$, гл. V, п. 101, теорема 1] или $[2$, гл. V,$\S 5.6])$ для любого $\sigma>0$ найдутся целые фунцкии $g_{\sigma, 1, r}$ и $g_{\sigma, 2, r}$ из класса $\mathbf{E}_{\sigma}$ такие, что

$$
\begin{aligned}
& \left|\varphi_{r}(x)-g_{\sigma, 1, r}(x)\right| \leqslant \frac{K_{r}}{\sigma^{r}} \quad \forall x \in \mathbb{R}, \\
& \left|\psi_{r}(x)-g_{\sigma, 2, r}(x)\right| \leqslant \frac{\gamma_{1}(r) K_{r+1}}{\sigma^{r+1}} \quad \forall x \in \mathbb{R} .
\end{aligned}
$$

Поскольку $x^{r} \varphi_{r}(x)$ и $x^{r-1} \psi_{r}(x)$ - четные функции, то $x^{r} g_{\sigma, 1, r}(x)$ и $x^{r-1} g_{\sigma, 2, r}(x)$ также можно считать четньми.

Теперь из представления (27) следует, что

$$
\left|F\left(x^{2}\right)-(2 x)^{r} g_{\sigma, 1, r}(x)-(2 x)^{r-1} g_{\sigma, 2, r}(x)\right| \leqslant \frac{2^{r} K_{r}}{\sigma^{r}} x^{r}+\frac{\gamma(r)}{\sigma^{r+1}} x^{r-1} \quad \forall x \in \mathbb{R},
$$


где $\gamma(r)=\gamma_{1}(r) 2^{r-1} K_{r+1}$.

Обозначим $G_{\sigma, r}(x)=(2 x)^{r} g_{\sigma, 1, r}(x)+(2 x)^{r-1} g_{\sigma, 2, r}(x)$. Тогда функция $G_{\sigma, r} \in \mathbf{E}_{\sigma}$ и четная. Заменяя в $(28) x^{2}$ на $x$ и возвращаясь к функции $f$, получим неравенство (1) с функцией

$$
g(x)=G_{\sigma, r}(\sqrt{x})+\sum_{k=0}^{r} \frac{1}{k !} f^{(k)}(0) x^{k} \in \mathbf{H}_{\sigma} .
$$

Предположим теперь, что в (1) при некотором $r \in \mathbb{N}$ вместо $2^{r} K_{r}$ в главном члене можно поставить некоторую константу $A_{r}$. Для $\varepsilon \in(0,1)$ положим $\sigma_{\varepsilon}=[\sigma]+1-\varepsilon$, тог да $\left[\sigma_{\varepsilon}\right]=[\sigma]$.

Рассмотрим функции $\Phi_{\sigma, r}$ из леммы 5 . Легко видеть, что они удовлетворяют условиям леммы 4 и в силу их $2 \pi$-периодичности

$$
\mathscr{A}_{\lambda}\left[\Phi_{\sigma, r}\right]=\mathscr{A}_{[\lambda]}\left[\Phi_{\sigma, r}\right] \quad \forall \lambda>0
$$

(см. [2, гл. II, $\S 2.6$, п. 2.6.21]). Применяя к функции $\Phi_{\sigma, r}$ лемму 4 с $\sigma_{\varepsilon}$ вместо $\sigma$, из $(29)$ и леммы 5 имеем

$$
\frac{A_{r}}{2^{r} \sigma_{\varepsilon}^{r}} \geqslant \mathscr{A}_{\sigma_{\varepsilon}}\left[\Phi_{\sigma, r}\right]=\mathscr{A}_{\left[\sigma_{\varepsilon}\right]}\left[\Phi_{\sigma, r}\right]=\mathscr{A}_{[\sigma]}\left[\Phi_{\sigma, r}\right]=\mathscr{A}_{\sigma}\left[\Phi_{\sigma, r}\right]=\frac{K_{r}}{([\sigma]+1)^{r}} .
$$

Таким образом,

$$
A_{r} \geqslant \frac{2^{r} K_{r} \sigma_{\varepsilon}^{r}}{([\sigma]+1)^{r}}
$$

Переходя в этом неравенстве к пределу при $\varepsilon \rightarrow+0$, получаем $A_{r} \geqslant 2^{r} K_{r}$, что и требовалось доказать.

\section{СПИСОК ЦИТИРОВАННОЙ ЛИТЕРАТУРЫ}

[1] Ахиезер Н. И. Лекции по теории аппроксимации. М.: Наука, 1965.

[2] Тиман А.Ф. Теория приближения функций действительного переменного. М.: Физматгиз, 1960.

[3] Корнейчук Н. П. Точные константы в теории приближения. М.: Наука, 1987.

[4] Ибрагимов И. И. Теория приближения целыми функциями. Баку: Элм, 1979.

[5] Бернштейн С. Н.Функции конечной степени и функции конечной полустепени. // Собрание сочинений. Т. 2. М.: АН СССР, 1954. С. 479-492.

[6] Брудный Ю. А. Приближение целыми функциями на внешности отрезка и полуоси // Изв. АН СССР. Сер. матем. 1959. Т. 23. № 4. C. 595-612.

[7] Никольский С. М. О наилучшем приближении многочленами функций, удовлетворяющих условию Липшица // Изв. АН СССР. Сер. матем. 1946. Т. 10. № 4. С. 295-318.

[8] Корнейчук Н.П., Половина А.И. О приближении непрерывных дифференцируемых функций алгебраическими полиномами на отрезке // Докл. АН СССР. 1966. Т. 166. № 2. C. $281-283$.

[9] Темляков В. Н. Приближение функций из класса $W_{\infty}^{1}$ алгебраическими многочленами // Матем. заметки. 1981. Т. 29. № 4. С. 597-602.

[10] Тригуб Р.М. Прямые теоремы о приближении алгебраическими полиномами гладких функций на отрезке // Матем. заметки. 1993. Т. 54. №6. С. 113-121.

[11] Бернштейн С. Н. О наилучшем приближении на всей вещественной оси при помощи целых функций данной степени, 3 (1946) // Собрание сочинений. Т. 2. М.: АН СССР, 1954. C. $379-383$. 